\title{
Semantic Learning Modifies Perceptual Face Processing
}

\author{
Jennifer J. Heisz and Judith M. Shedden
}

\begin{abstract}
Face processing changes when a face is learned with personally relevant information. In a five-day learning paradigm, faces were presented with rich semantic stories that conveyed personal information about the faces. Event-related potentials were recorded before and after learning during a passive viewing task. When faces were novel, we observed the expected N170 repetition effect—a reduction in amplitude following face repetition. However, when faces were learned with personal information, the N170 repetition effect was eliminated, suggesting that semantic information modulates the N170 repetition effect. To control for the possibility that a simple perceptual effect contributed to the change in the N170 repetition effect, another experiment was conducted using
\end{abstract}

\section{INTRODUCTION}

Many differences exist in our recognition of familiar and unfamiliar faces. Familiar faces are recognized faster and more accurately than unfamiliar faces (Burton, Wilson, Cowen, \& Bruce, 1999; Klatzky \& Forrest, 1984; Bruce, 1982; Ellis, Shepherd, \& Davies, 1979). We rely on different facial information to identify familiar and unfamiliar faces; when identifying familiar faces, we tend to focus on the eye and eye region, whereas when identifying unfamiliar faces, we tend to scan the entire face image (Heisz \& Shore, 2008; Althoff \& Cohen, 1999). These differences have led many to theorize that different neurological processes subserve familiar and unfamiliar face recognition. In the following experiments, we investigate differences in familiar and unfamiliar face recognition processing as reflected in the repetition effect of the event-related potential (ERP) N170 component.

The N170 is a negative-going component that peaks approximately $170 \mathrm{msec}$ poststimulus onset. It is maximally recorded over the occipito-temporal cortex and is typically larger over the right hemisphere than the left hemisphere. Mainly implicated in perceptual (physical; bottom-up) face processing, the amplitude of the N170 is larger for faces than nonface objects (Carmel \& Bentin, 2002; Sagiv \& Bentin, 2001; Eimer, 2000a; George,

McMaster University, Hamilton, Ontario, Canada stories that were not related to the person (i.e., stories about rocks and volcanoes). Although viewers were exposed to the faces an equal amount of time, the typical N170 repetition effect was observed, indicating that personal semantic information associated with a face, and not simply perceptual exposure, produced the observed reduction in the N170 repetition effect. These results are the first to reveal a critical perceptual change in face processing as a result of learning person-related information. The results have important implications for researchers studying face processing, as well as learning and memory in general, as they demonstrate that perceptual information alone is not enough to establish familiarity akin to real-world person learning.
Evans, Fiori, Davidoff, \& Renault, 1997; Bentin, Allison, Puce, Perez, \& McCarthy, 1996) and is larger for whole facial images compared to facial images lacking internal features (e.g., eyes, nose, mouth) or external features (e.g., head contour, hair) (Eimer, 2000a). Although the N170 is sensitive to both facial features and facial configuration (i.e., the spatial relations among the features of a face) (Bentin, Golland, Flevaris, Robertson, \& Moscovitch, 2006; Boutsen, Humphreys, Praamstra, \& Warbrick, 2006; Caharel, Fiori, Bernard, Lalonde, \& Rebai, 2006; Itier, Latinus, \& Taylor, 2006; Latinus \& Taylor, 2005, 2006; Carbon, Schweinberger, Kaufmann, \& Leder, 2005; Schyns, Jentzsch, Johnson, Schweinberger, \& Gosselin, 2003), the right hemisphere is particularly sensitive to changes in facial configuration, whereas the left hemisphere is particularly sensitive to changes in facial features (Scott \& Nelson, 2006).

Facial identity processing is revealed through the N170 repetition effect. Characterized by a reduction in the N170 amplitude following immediate face presentations (Heisz, Watter, \& Shedden, 2006a, 2006b; Itier \& Taylor, 2002, 2004; Guillaume \& Tiberghien, 2001; Campanella et al., 2000; George, Jemel, Fiori, \& Renault, 1997), the N170 repetition effect is observed for repeated presentation of the same image (Heisz et al., 2006a, 2006b; Itier \& Taylor, 2002, 2004; George, Jemel, et al., 1997), as well as successive presentation of two images portraying the same individual with different 
facial expressions (Guillaume \& Tiberghien, 2001), different background images (Guillaume \& Tiberghien, 2001), or completely different physical images (Campanella et al., 2000), suggesting that the effect cannot be explained as perceptual habituation. Importantly, N170 repetition effects are only observed for unfamiliar faces. The N170 repetition effect is not observed for highly familiar or famous faces (Schweinberger, Pickering, Burton, \& Kaufmann, 2002; Schweinberger, Pickering, Jentzsch, Burton, \& Kaufmann, 2002). Caharel, Poiroux, and Bernard (2002) demonstrated this dissociation in a passive viewing task in which observers viewed 100 repetitions of one's own face, a famous face, and an unfamiliar face. The N170 amplitude response in the right hemisphere was attenuated for repetitions of an unfamiliar face only; the attenuation did not occur for repetitions of one's own face and repetitions of a famous face. Similarly, another study reported repetition effects for unfamiliar, and not for famous faces (Henson et al., 2003).

A critical difference between familiar and unfamiliar faces is the amount of person-specific information associated with the face. Highly familiar faces are associated with a rich network of person-specific information, whereas unfamiliar faces are associated with little or no information. According to the current face recognition model, person-specific information aids in our identification of a particular individual (Burton, Bruce, \& Hancock, 1999; Burton, Bruce, \& Johnston, 1990; Bruce \& Young, 1986). Different aspects of a person can be used to retrieve relevant information, resulting in superior memory for highly familiar individuals. Unfamiliar faces do not afford such recognition benefits because they lack associated information, resulting in recognition based primarily on perceptual processing. We hypothesize that perceptual identity processing is reflected in the N170 repetition effect, which is observed for unfamiliar faces because perceptual processing is the primary mode of recognition. In contrast, the N170 repetition effect is not observed for familiar faces because both perceptual processing and semantic processing support recognition. Indeed, other top-down processes (e.g., expectations, attention) modify processing at the N170 by activating facial representations (Jemel, Pisani, Calabria, Crommelinck, \& Bruyer, 2003; Bentin, Sagiv, Mecklinger, Friederici, \& von Crammon, 2002; Eimer, 2000b). Accordingly, the $\mathrm{N} 170$ repetition effect elicited by an unfamiliar face should be reduced or eliminated after acquiring person-specific information.

In the present experiments, we used a 5-day learning paradigm to examine the influence of face learning on the N170 repetition effect. In a between-subjects design, one group of subjects learned faces with related stories (i.e., stories that conveyed person-specific information) and another group of subjects learned faces with unrelated stories (i.e., stories about rocks and other inanimate objects). ERPs were recorded before and after learning. Prior to learning when all faces were unfamiliar, we expected all faces to elicit the N170 repetition effect because perceptual processing alone would support recognition. After learning faces with related stories, we expected a reduction of the N170 repetition effect because both perceptual and semantic processing would support recognition. In contrast, after learning faces with unrelated stories, we expected to observe the N170 repetition effect because recognition would remain supported by perceptual processing.

\section{METHODS}

\section{Subjects}

Twenty volunteers from the McMaster University community participated in the experiment, 10 volunteers were randomly assigned to learn faces with related stories $(5$ men, mean age $=20.6$ years, 2 left-handed) and 10 volunteers were randomly assigned to learn faces with unrelated stories ( 3 men, mean age $=20.1$ years, 2 left-handed). All subjects reported normal or correctedto-normal vision. Informed consent was obtained from each observer. Eligible observers received course credit plus $\$ 15.00$ for their participation, and the remainder received $\$ 40.00$ compensation. All procedures complied with the tricouncil policy on ethics (Canada) and were approved by the McMaster Ethics Research Board.

\section{Stimuli}

Stimulus presentation and manual response measurement were controlled by Presentation experimental software (Version 9.9, www.neuro-bs.com), running on a Pentium 4 Computer under Windows XP operating system. The stimuli were displayed on a 17-in. color CRT display at a resolution of $1280 \times 1024$ and a frame refresh rate of $85 \mathrm{~Hz}$. Participants were seated $80 \mathrm{~cm}$ from the display and the experiment was run in a dimly lit room.

The face stimuli consisted of 10 colored images of Caucasian individuals ( 5 men) with neutral expressions. Faces were without glasses and a black wrap concealed clothing. Faces were adopted from a larger set provided by Dr. Daphne Maurer's Visual Developmental Lab, Department of Psychology, Neuroscience and Behaviour, McMaster University (Geldart, Maurer, \& Henderson, 1999). Facial images were presented at the center of the display on a gray background, approximately $4.8^{\circ}$ of visual angle wide and $6.0^{\circ}$ of visual angle high. The average luminance value for each face image was approximately $19.7 \mathrm{~cd} / \mathrm{m}^{2}$. Eight images ( 4 males, 4 females) were selected per subject and assigned to a particular condition; the assignment of face stimuli to particular conditions was counterbalanced across subjects.

The story stimuli consisted of related stories and unrelated stories. P. Darren Parker, a graduate of Television 
Production and Writing, Humber College, composed the related stories. The related stories captured "A-week-inthe-life-of" four unique and interconnected characters. The stories were divided into five sections of approximately equivalent length $(\sim 4.5 \mathrm{~min})$ and narrated to subjects in chronological order over five consecutive days. Each section represented "A-day-in-the-life-of" the four characters, conveying new information about them while building on information from previous sections. Whenever a specific character appeared in the narrated story, that character's face was presented visually. In total, two characters appeared in the story approximately 21 min each (main characters), and two characters appeared in the story approximately 3 min each (sub characters). The unrelated stories were created from compilation of geographical articles about various inanimate objects, such as rocks and volcanoes. The articles were randomly assigned to one of five sections with approximately equivalent length $(\sim 4.5 \mathrm{~min})$ and narrated to subjects over five consecutive days. Each section presented new information that was completely unrelated to the information from previous sections. As in the related story condition, two faces were presented for approximately $21 \mathrm{~min}$ each and two faces were presented for approximately $3 \mathrm{~min}$ each, so that both groups were exposed to the faces at the same time and for the same duration; the content of the stories was the only difference between the two groups.

The story stimuli were recorded in a sound attenuated room, with Shure Professional head-worn Dynamic Microphone and Marantz Professional CD Recorder. Audacity editing suite was used to segment the audiofiles, minimize noise, and equate loudness across each separate wavefile. The same female voice was used to create all auditory stimuli. Auditory stimuli were presented using Labtec remote computer speakers mounted in front of the participant, presented at approximately $60 \mathrm{~dB}$ or the level of a normal conversation.

\section{Procedure}

The experiment was conducted over five consecutive days. In a between-subjects design, one group of subjects learned faces with related stories (i.e., stories that conveyed person-specific information) and the other group of subjects learned faces with unrelated stories (i.e., stories about rocks and other inanimate objects). During the learning sessions, a face was presented at the center of the display and subjects were instructed to keep their eyes on the face image; eye movements were recorded to ensure this. While viewing the face image, subjects in the related story condition listened to related stories about the individual and subjects in the unrelated story condition listened to stories about rocks and other inanimate objects. For the group that learned faces with related stories, the face image presented on the display always corresponded with the character being described in the story. When the story shifted to another character, the new character's face replaced the previous character's face, allowing the visual face image and auditory character-related story to be associated for each character. The group that learned faces with unrelated stories saw the same sequence of faces (with the same stimulus onset times and durations for each face), but in this condition, the faces did not relate to the content of the story. In other words, we presented both groups with identical visual stimuli and varied the auditory stimuli, such that one group heard person-related stories characterizing the faces and the other group heard unrelated stories about rocks and other inanimate objects. Following each learning session, subjects answered questions about the story content to encourage them to pay attention to the stories. Approximately 2 months after the learning session, we tested 8 of the 10 subjects from the related story condition for their memory of the faces; each subject was $100 \%$ correct at naming the characters' faces.

ERPs were recorded before and after learning. Each ERP session lasted approximately $1 \mathrm{hr}$. The prelearning ERP session consisted of six face stimuli: four learned faces (i.e., faces presented during the learning session) and two control faces (i.e., faces presented in ERP sessions only). The postlearning ERP session consisted of eight stimuli, four learned faces, and four control faces (i.e., the 2 control faces from the prelearning ERP session plus 2 new control faces). Faces were presented for $750 \mathrm{msec}$ followed by an interstimulus interval of $750 \mathrm{msec}$, during which a fixation point was presented. Faces were presented in pseudorandomized order; each face was presented approximately 200 times, half of which were immediate repetitions. Subjects performed a passive viewing task in which they were instructed to consider each face's identity. This passive viewing paradigm was adopted from Caharel et al. (2002).

\section{Electrophysiology}

The ActiveTwo BioSemi electrode system was used to record continuous electroencephalographic (EEG) activity from $128 \mathrm{Ag} / \mathrm{AgCl}$ scalp electrodes plus 4 addition electrodes placed at the outer canthi and just below each eye for recording horizontal and vertical eye movements. Two additional electrodes, common mode sense (CMS) active electrode and driven right leg (DRL) passive electrode, were also used. These electrodes replace the "ground" electrodes used in conventional systems (www.biosemi.com/faq/cms\&drl.htm). Because the BioSemi system is an active electrode system, there is no conventional reference electrode; a monopolar signal is stored for each active electrode and all rereferencing is done in software after acquisition. The continuous signal was acquired with an open band pass from DC to $150 \mathrm{~Hz}$ and digitized at $512 \mathrm{~Hz}$. The signal was band-pass filtered off-line at 0.1 to $30 \mathrm{~Hz}$ and rereferenced to a common average reference. 
ERP averaging and analysis were performed using EEProbe software (ANT; www.antsoftware.nl). EEG and EOG artifacts were removed using a $\pm 35-\mu \mathrm{V}$ deviation over 200-msec intervals on all electrodes. Blink artifacts were selected manually and corrected by a subtraction of VEOG propagation factors via a regression algorithm on EOG components (using EEProbe signal processing software). A 1000-msec recorded EEG epoch, including $100 \mathrm{msec}$ prestimulus baseline and $900 \mathrm{msec}$ interval following stimulus onset, was chosen for ERP averaging. ERP waveforms were then averaged separately for each electrode for each experimental condition.

\section{Data Analysis}

ERP analysis of the N170 component focused on an occipito-temporal region of interest, which exhibited maximal N170 amplitude. Over left and right hemispheres, a set of four adjacent electrode pairs was examined, forming a cluster around P7/P8 (also called T5/T6 in the 10-20 system), such that the distance between the adjacent electrodes was $2 \mathrm{~cm}$ (center to center) posterior and inferior to $\mathrm{P} 7 / \mathrm{P} 8$. This is the electrode location most commonly associated with N170 effects in the literature (e.g., Rossion \& Jacques, 2008; Itier et al., 2006) and displayed the largest peak-to-peak amplitude between P1 and N170 $(\sim 6 \mu \mathrm{V})$. For each subject, the pair from this cluster that showed maximal N170 effects was selected for analysis. The amplitude of the N170 component was isolated using a time window ranging from 120 to $200 \mathrm{msec}$, obtained via inspection of the grand-average waveforms. Repeated measures analysis of variance (ANOVA) was conducted for N170 peak amplitude in a five-way design with one between-subject factor of group (faces learned with related stories, faces learned with unrelated stories) and four within-subject factors of session (prelearning, postlearning), hemisphere (right, left), face type (learned, control), and repetition (preceeded by the same face, preceded by a different face).

\section{RESULTS}

Initial statistical analyses were conducted to determine whether there were any differences between responses to main and sub character faces, and between responses to the different control faces. Repeated measures ANOVA tests revealed no main effects or interactions of main versus sub character faces with factors of session and repetition (all $F \mathrm{~s}<0.7$ ). Separate repeated measures ANOVA showed no main effects or interactions between the different control faces with factors of session and repetition (all FS $<0.8$ ). Therefore, all subsequent analyses collapsed over individual faces and focused on two categories of faces: learned faces (presented during the ERP sessions and during the 5 days of stories) and control faces (presented only during the ERP sessions).
Two figures illustrate the results. Figure 1 depicts grand-average waveforms for the N170 ERP component elicited in the right versus left hemispheres during prelearning versus postlearning sessions. Learned faces and control faces are compared between subjects in the related versus unrelated story conditions, and the N170 repetition effect is reflected in the amplitude differences for faces preceded by the same face image versus faces preceded by a different face image. Figure 2 depicts the difference in left and right hemisphere N170 repetition effect between pre- and postlearning sessions for learned and control faces in the related and unrelated story conditions (e.g., subtract the postlearning repetition effect from the prelearning repetition effect for each condition). A positive value indicates a reduction in the N170 repetition effect following learning, whereas a negative value indicates an enhancement in the N170 repetition effect following learning.

Based on a significant five-way interaction $[F(1,18)=$ $5.271, p<.05]$, separate analyses were conducted to focus on key issues. The first set of analyses involved two repeated measures ANOVA tests carried out separately for the related and unrelated stories groups.

Faces learned with unrelated stories revealed a simple main effect of repetition $[F(1,9)=22.327, p<.01]$, such that N170 amplitudes were reduced for faces preceded by the same face compared to faces preceded by a different face. Importantly, this repetition effect did not interact with session. No other main effects or interactions were observed in the unrelated stories condition, and the remaining analyses focus on the related stories group.

Faces learned with related stories revealed a significant four-way interaction of session, hemisphere, face type, and repetition $[F(1,9)=6.621, p<.05]$. We further examined the repetition effect by collapsing the data in two different sets of analyses. First, we analyzed the prelearning and postlearning sessions separately using a three-factor (Hemisphere $\times$ Face type $\times$ Repetition) repeated measures ANOVA. This allowed us to ask directly whether the repetition effect differed between learned and control faces within each of the two ERP sessions. The prelearning session revealed a simple main effect of repetition $[F(1,9)=10.177, p<.05]$, such that N170 amplitudes were reduced for faces preceded by the same face compared to faces preceded by a different face. Importantly, this effect did not interact with face type, showing that both learned and control faces produced the same repetition effects in the prelearning session. No other main effects or interactions were observed in the prelearning session. The postlearning session revealed a simple main effect of repetition $[F(1,9)=76.976, p<.001]$, and a significant two-way interaction between face type and repetition $[F(1,9)=$ $5.635, p<.05]$, such that N170 repetition effects were smaller for learned faces compared to control faces $[t(9)=$ $-2.374, p<.05]$. This result supports the hypothesis that 


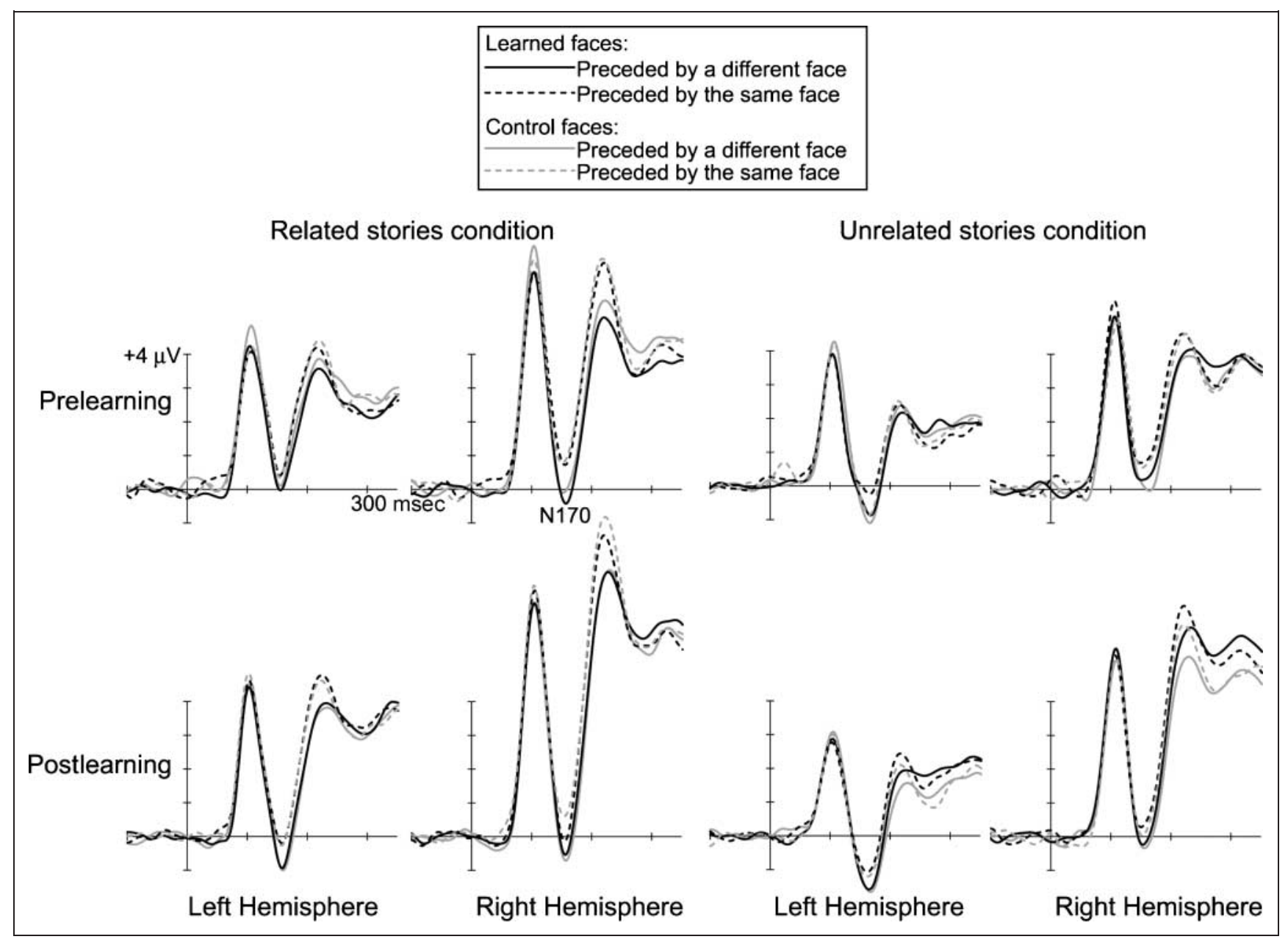

Figure 1. Grand-average waveforms for the N170 ERP component measured at P7/P8 (also called T5/T6) during prelearning versus postlearning sessions. Learned faces and control faces are compared between subjects in the related versus unrelated story conditions, and the N170 repetition effect is reflected in the amplitude differences for faces preceded by the same face image versus faces preceded by a different face image.

the repetition effect for learned and control faces differs following the 5 days of related stories about the learned faces.

Second, we analyzed the learned and control faces separately using a three-factor (Hemisphere $\times$ Session $\times$ Repetition) repeated measures ANOVA to directly compare the shift in the N170 repetition effect across the pre- and postlearning sessions. There was a main effect of session for both analyses due to larger amplitudes overall in the postlearning session compared to the prelearning session [learned faces: $F(1,9)=11.341, p<.01$; control faces: $F(1,9)=6.414, p<.05]$. The control faces revealed a simple main effect of repetition $[F(1,9)=$ $50.033, p<.001]$, such that N170 amplitudes were reduced for faces preceded by the same face compared to faces preceded by a different face. Importantly, the repetition effect for the control faces did not interact with session. No other main effects or interactions were observed for the control faces.

Learned faces revealed a simple main effect of repetition $[F(1,9)=19.003, p<.01]$, and a three-way in- teraction between session, hemisphere, and repetition $[F(1,9)=5.094, p=.05]$. For the prelearning session, repetition effects were observed in both right $[t(9)=$ $-3.640, p=.005]$ and left $[t(9)=-2.414, p=.039]$ hemispheres. However, during the postlearning session, repetition effects were only observed in the left hemisphere $[t(9)=-3.629, p=.005]$. The N170 repetition effect was not observed for learned faces in the right hemisphere $[t(9)=-1.458, p=n s]$. In other words, the N170 repetition effect, which was largest over the right hemisphere in the prelearning session, was eliminated over the right hemisphere after learning faces with related stories.

\section{DISCUSSION}

We examined the influence of face learning on perceptual face processing. Across five consecutive days, subjects viewed previously novel faces while either listening to stories about the individuals or listening to unrelated stories (i.e., stories about rocks and other inanimate 


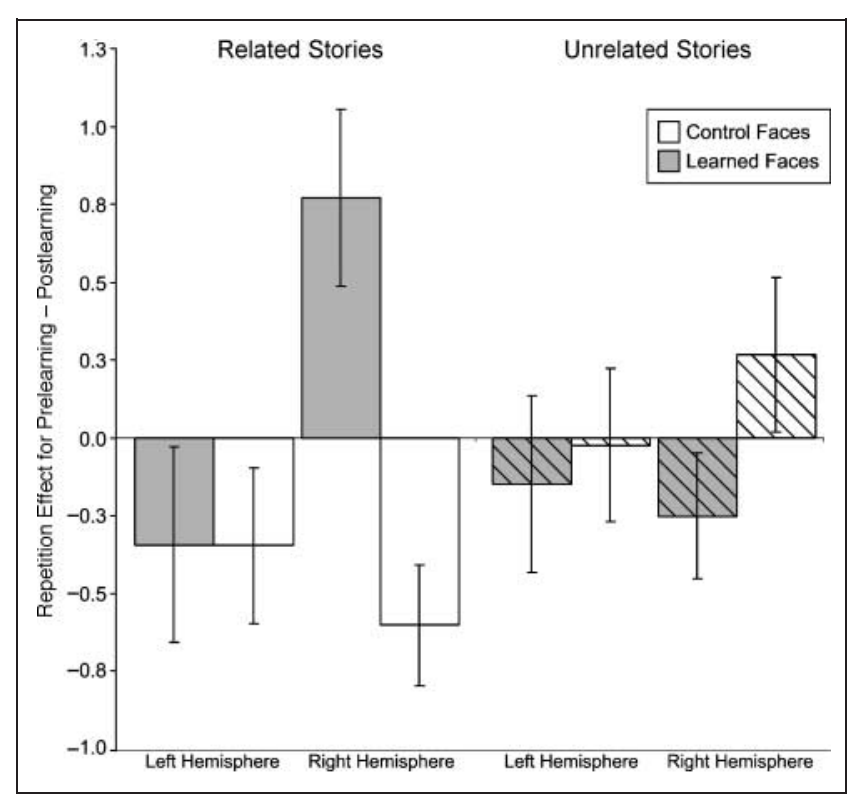

Figure 2. The difference in left and right hemisphere N170 repetition effect between pre- and postlearning sessions for learned and control faces in the related and unrelated story conditions (e.g., subtract the postlearning repetition effect from the prelearning repetition effect for each condition). A positive value indicates a reduction in the N170 repetition effect following learning, whereas a negative value indicates an enhancement in the N170 repetition effect following learning. The right hemisphere N170 repetition effect was reduced after learning faces with related stories. Error bars reflect standard error.

objects). ERPs were recorded before and after learning, and we were interested in learning-induced changes in the N170, an ERP component primarily implicated in perceptual face processing (Carmel \& Bentin, 2002; Sagiv \& Bentin, 2001; Eimer, 2000b; Bentin et al., 1996). We expected a change in the N170 repetition effect, which is characterized by a reduction in the N170 amplitude following immediate presentations of the same face (Heisz et al., 2006a, 2006b; Itier \& Taylor, 2002, 2004; Guillaume \& Tiberghien, 2001; Campanella et al., 2000; George, Evans, et al., 1997; George, Jemel, et al., 1997). The N170 repetition effect is observed for unfamiliar faces but not familiar faces (Henson et al., 2003; Caharel et al., 2002; Schweinberger, Pickering, Burton, et al., 2002; Schweinberger, Pickering, Jentzsch, et al., 2002), therefore we expected the repetition effect to be reduced or eliminated following face learning. We hypothesized that the N170 repetition effect reflects perceptual identity processing as the primary mode used to recognizing unfamiliar faces. In contrast, processing of familiar faces is further supported by semantic knowledge about the person, which leads to identity processing at a later stage, thus eliminating the N170 repetition effect for familiar faces. To test this, we compared the N170 repetition effect elicited after learning faces with semantically related stories versus learning faces with unrelated stories. As predicted, the N170 repetition effect was affected by as little as 5 days of experience with a par- ticular face. The N170 repetition effect was eliminated by associating previously novel faces with a rich semantic context in the form of stories, whereas no change was observed in the N170 repetition effect after learning faces with unrelated stories.

Previous studies using famous faces reported comparable lack of the N170 repetition effect (Henson et al., 2003; Caharel et al., 2002; Schweinberger, Pickering, Burton, et al., 2002; Schweinberger, Pickering, Jentzsch, et al., 2002) and when unfamiliar faces were used in similar paradigms the N170 repetition effect was observed (Heisz et al., 2006a, 2006b; Itier \& Taylor, 2002, 2004; Guillaume \& Tiberghien, 2001; Campanella et al., 2000; George, Jemel, et al., 1997). In the present study, prior to face learning, all faces were unfamiliar and elicited the N170 repetition effect. After a mere 5 days of learning the faces with related stories, the N170 repetition effect was eliminated over the right hemisphere (P8), mimicking that observed for famous faces. These results suggest that perceptual processing as reflected by the N170 repetition effect is modulated by familiarity.

Some studies fail to show any N170 repetition effect for familiar faces (Henson et al., 2003; Schweinberger, Pickering, Jentzsch, et al., 2002; Eimer, 2000a), yet we continue to show the N170 repetition effect at P7 (left hemisphere) for the related story faces. One possibility for this difference may be that we are observing a transition from unfamiliar to familiar face processing, which may require more experience to reach the level of familiarity of the well-known faces used in these other studies, and that this transition from unfamiliar to familiar begins with processing changes in right hemisphere face areas. Indeed, the left hemisphere represents faces in an image-independent manner (Cooper, Harvey, Lavidor \& Schweinberger, 2007), a form of facial representation supported by a high level of familiarity (Eger, Schweinberger, Dolan, \& Henson, 2005). Our results suggest a special role for the right hemisphere in acquisition of face familiarity.

Although personal information was acquired for faces learned with related stories, the simple visual repetition of faces over the 5 days may have been an important factor in the elimination of the N170 repetition effect. Previous studies using famous faces to investigating the N170 repetition effect were unable to control for this important variable (Henson et al., 2003; Caharel et al., 2002; Schweinberger, Pickering, Burton, et al., 2002; Schweinberger, Pickering, Jentzsch, et al., 2002). To determine the extent to which semantic knowledge and visual repetition were contributing to the change in the N170 repetition effect, we repeated the experiment using stories that were not related to the person; across five consecutive days, subjects viewed the faces while listening to stories about rocks and other inanimate objects. This created a situation in which faces were familiarized through simple visual repetition, but were not personally related to any semantic information. Unlike 
learning faces with related stories, learning faces with unrelated stories caused no change in the N170 repetition effect. Although the faces in both learning paradigms were seen equally for 5 days, it was the person-related semantic information associated with the faces, and not simply the visual repetition, that produced the reduction in the N170 repetition effect. In other words, knowing information about a person changed the way their face was processed at a perceptual level.

Our results fit well with the previous studies demonstrating behavioral differences between familiar and unfamiliar faces processing. Familiar faces are processed faster and more accurately than unfamiliar faces (Burton, Bruce, et al., 1999; Burton, Wilson, et al., 1999; Klatzky \& Forrest, 1984; Bruce, 1982; Ellis et al., 1979). Moreover, we rely on different facial information to identify familiar and unfamiliar faces, focusing more on the eyes and eye region when identifying familiar faces and scanning the entire face image when identifying unfamiliar faces (Heisz \& Shore, 2008; Althoff \& Cohen, 1999). These processing differences are consistent with the change in perceptual processing which we observe at the N170. Acquiring semantic knowledge associated with a face eliminated the N170 repetition effect. We purport this change is the result of top-down processes involving rich networks of semantic information that modulate perceptual processing of familiar faces, ultimately leading to a more robust representation of facial identity. Akin to the face recognition units of the face recognition model (Burton, Bruce, et al., 1999; Burton et al., 1990; Bruce \& Young, 1986), the N170 may be the premier stage in which face processing depends on familiarity. Although this is earlier than previously reported (e.g., Joyce \& Kutas, 2005), our data suggest that the perceptual stage of face processing as reflected by the N170 depends on the existence of person-specific semantic information associated with the particular face.

In conclusion, associating semantic information with a previously novel face changes the way we process that face at a perceptual level. This is the first article to demonstrate a dynamic change from novel to familiar, adding critical understanding to the processing changes in face recognition following learning. This article highlights the importance of the learning paradigm used to induce familiarization; familiarization achieved through simple visual repetition of a face is not sufficient to change the N170 repetition effect. However, familiarization achieved through visual repetition and rich semantic stories about the face is sufficient to bring about the change in the N170 repetition effect. The latter is more comparable to real-world person learning.

\section{Acknowledgments}

We thank Dr. Daphne Maurer and the Vision Development Lab at McMaster University's Department of Psychology, Neuroscience \& Behaviour, for use of their face photographs, from which the stimuli were constructed. This research was supported by Natural Sciences and Engineering Research Council of Canada (NSERC), Grant \#170353 to Judith M. Shedden, and NSERC Canadian Graduate Scholarship-Doctorate to Jennifer J. Heisz.

Reprint requests should be sent to Judith M. Shedden or Jennifer J. Heisz, Department of Psychology, Neuroscience \& Behaviour, McMaster University, 1280 Main Street West, Hamilton, Ontario, Canada L8S 4K1, or via e-mail: shedden@mcmaster.ca; heiszjj@mcmaster.ca.

\section{REFERENCES}

Althoff, R. R., \& Cohen, N. J. (1999). Eye-movement based memory effect: A reprocessing effect in face perception. Journal of Experimental Psychology: Learning, Memory, and Cognition, 25, 997-1010.

Bentin, S., Allison, T., Puce, A., Perez, E., \& McCarthy, G. (1996). Electrophysiological studies of face perception in humans. Journal of Cognitive Neuroscience, 8, 551-565.

Bentin, S., Golland, Y., Flevaris, A., Robertson, L. C., \& Moscovitch, M. (2006). Processing the trees and the forest during initial stages of face perception: Electrophysiological evidence. Journal of Cognitive Neuroscience, 18, 1406-1421.

Bentin, S., Sagiv, N., Mecklinger, A., Friederici, A., \& von Crammon, Y. D. (2002). Priming visual face-processing mechanisms: Electrophysiological evidence. Psychological Science, 13, 190-193.

Boutsen, L., Humphreys, G. W., Praamstra, P., \& Warbrick, T. (2006). Comparing neural correlates of configural processing in faces and objects: An ERP study of the Thatcher illusion. Neuroimage, 32, 352-367.

Bruce, V. (1982). Changing faces-Visual and non-visual coding processes in face recognition. British Journal of Psychology, 73, 105-116.

Bruce, V., \& Young, A. (1986). Understanding face recognition. British Journal of Psychology, 77, 305-327.

Burton, A. M., Bruce, V., \& Hancock, P. J. B. (1999). From pixels to people: A model of familiar face recognition. Cognitive Science, 23, 1-31.

Burton, A. M., Bruce, V., \& Johnston, R. A. (1990). Understanding face recognition with an interactive activation model. British Journal of Psychology, 81, 361-380.

Burton, A. M., Wilson, S., Cowen, M., \& Bruce, V. (1999). Face recognition in poor quality video: Evidence from security surveillance. Psychological Science, 10, 243-248.

Caharel, S., Fiori, N., Bernard, C., Lalonde, R., \& Rebai, M. (2006). The effect of inversion and eye-displacement of familiar and unknown faces on early and late-stage ERPs. International Journal of Psychophysiology, 62, 141-151.

Caharel, S., Poiroux, S., \& Bernard, C. (2002). ERPs associated with familiarity and the degree of familiarity during face recognition. International Journal of Neuroscience, 112, 1531-1544.

Campanella, S., Hanoteau, C., Depy, D., Rossion, B., Bruyer, R., Crommelinck, M., et al. (2000). Right modulation in a face discrimination task: An account for categorical perception of familiar faces. Psychophysiology, 37, 796-806.

Carbon, C. C., Schweinberger, S. R., Kaufmann, J. M., \& Leder, H. (2005). The Thatcher Illusion seen by the brain: An event-related brain potentials study. Cognitive Brain Research, 24, 544-555.

Carmel, D., \& Bentin, S. (2002). Domain specificity versus expertise: Factors influencing distinct processing of faces. Cognition, 83, 1-29. 
Cooper, T. J., Harvey, M., Lavidor, M., \& Schweinberger, S. R. (2007). Hemispheric asymmetries in image-specific and abstractive priming of famous faces: Evidence from reaction times and event-related brain potentials. Neuropsychologia, 45, 2910-2921.

Eger, E., Schweinberger, S. R., Dolan, R. J., \& Henson, R. N. (2005). Familiarity enhances invariance of face representations in human ventral visual cortex: fMRI evidence. Neuroimage, 26, 1128-1139.

Eimer, M. (2000a). Event-related brain potentials distinguish processing stages involved in face perception and recognition. Clinical Neurophysiology, 111, 694-705.

Eimer, M. (2000b). Attention modulation of event-related brain potentials sensitive to faces. Cognitive Neuropsychology, 17, $103-116$

Ellis, H. D., Shepherd, J. W., \& Davies, G. M. (1979). Identification of familiar and unfamiliar faces from internal and external features: Some implication for theories of face recognition. Perception, 105, 482-498.

Geldart, S., Maurer, D., \& Henderson, H. (1999). Effects of the height of the internal features of faces on adults' aesthetic ratings and 5-month-olds' looking times. Perception, 28, 839-850.

George, N., Evans, J., Fiori, N., Davidoff, J., \& Renault, B. (1997). Brain events related to normal and moderately scrambled faces. Cognitive Brain Research, 4, 65-76.

George, N., Jemel, B., Fiori, J., \& Renault, B. (1997). Race and shape repetition effects in humans: A spatio-temporal ERP study. NeuroReport, 8, 1417-1423.

Guillaume, F., \& Tiberghien, G. (2001). An event-related potential study of contextual modification in a face recognition task. NeuroReport, 12, 1209-1216.

Heisz, J. J., \& Shore, D. I. (2008). More efficient scanning for familiar face. Journal of Vision, 8, 1-10.

Heisz, J. J., Watter, S., \& Shedden, J. M. (2006a). Progressive N170 habituation to unattended repeated faces. Vision Research, 46, 47-56.

Heisz, J. J., Watter, S., \& Shedden, J. M. (2006b). Automatic face identity encoding at the N170. Vision Research, 46, 4604-4614

Henson, R. N., Goshen-Gottstein, Y., Ganel, T., Otten, L. J., Quayle, A., \& Rugg, M. D. (2003). Electrophysiological and haemodynamic correlates of face perception, recognition and priming. Cerebral Cortex, 13, 793-805.

Itier, R. J., Latinus, M., \& Taylor, M. J. (2006). Face, eye and object early processing: What is the face specificity? Neuroimage, 29, 667-676.
Itier, R. J., \& Taylor, M. J. (2002). Inversion and contrast polarity reversal affect both encoding and recognition processes of unfamiliar faces: A repetition study using ERPs. Neuroimage, 15, 353-372.

Itier, R. J., \& Taylor, M. J. (2004). Effects of repetition learning on upright, inverted and contrast-reversed face processing using ERPs. Neuroimage, 21, 1518-1532.

Jemel, B., Pisani, M., Calabria, M., Crommelinck, M., \& Bruyer, R. (2003). Is the N170 for faces cognitively penetrable? Evidence from repetition priming of Mooney faces of familiar and unfamiliar persons. Cognitive Brain Research, 17, 431-446.

Joyce, C. A., \& Kutas, M. (2005). Event-related potential correlates of long-term memory for briefly presented faces. Journal of Cognitive Neuroscience, 17, 757-767.

Klatzky, R. L., \& Forrest, F. H. (1984). Recognizing familiar and unfamiliar faces. Memory \& Cognition, 12, 60-70.

Latinus, M., \& Taylor, M. J. (2005). Holistic processing of faces: Learning effects with Mooney faces. Journal of Cognitive Neuroscience, 17, 1316-1327.

Latinus, M., \& Taylor, M. J. (2006). Face processing stages: Impact of difficulty and the separation of effects. Brain Research, 1123, 179-187.

Rossion, B., \& Jacques, C. (2008). Does physical interstimulus variance account for early electrophysiological face sensitive responses in the human brain? Ten lessons on the N170. Neuroimage, 39, 1959-1979.

Sagiv, N., \& Bentin, S. (2001). Structural encoding of human and schematic faces: Holistic and part-based processes. Journal of Cognitive Neuroscience, 13, 937-951.

Schweinberger, S. R., Pickering, E. C., Burton, A. M., \& Kaufmann, J. M. (2002). Human brain potential correlates of repetition priming in face and name recognition. Neuropsychologia, 40, 2057-2073.

Schweinberger, S. R., Pickering, E. C., Jentzsch, I., Burton, A. M., \& Kaufmann, J. M. (2002). Event-related brain potential evidence for a response of inferior temporal cortex to familiar face repetitions. Cognitive Brain Research, 14, 398-409.

Schyns, P. G., Jentzsch, I., Johnson, M., Schweinberger, S. R., \& Gosselin, F. (2003). A principled method for determining the functionality of brain responses. NeuroReport, 14, $1665-1669$.

Scott, L., \& Nelson, C. A. (2006). Featural and configural face processing in adults and infants: A behavioral and electrophysiological investigation. Perception, 35, 1107-1128. 